\title{
PREORDERS ON CANONICAL FAMILIES OF MODULES OF FINITE LENGTH
}

\author{
URI FIXMAN and FRANK OKOH
}

(Received 16 August 1990)

Communicated by $\mathbf{P}$. Schultz

\begin{abstract}
Let $R$ be an artinian ring. A family, $\mathscr{H}$, of isomorphism types of $R$-modules of finite length is said to be canonical if every $R$-module of finite length is a direct sum of modules whose isomorphism types are in $\mathscr{H}$. In this paper we show that $\mathscr{C}$ is canonical if the following conditions are simultaneously satisfied: (a) $\mathscr{H}$ contains the isomorphism type of every simple $R$-module; (b) $\mathscr{H}$ has a preorder with the property that every nonempty subfamily of $\mathscr{H}$ with a common bound on the lengths of its members has a smallest type; (c) if $M$ is a nonsplit extension of a module of isomorphism type $\mathrm{II}_{1}$ by a module of isomorphism type $\mathrm{II}_{2}$, with $\mathrm{II}_{1}, \mathrm{II}_{2}$ in $\mathscr{M}$, then $M$ contains a submodule whose type $\mathrm{II}_{3}$ is in $\mathscr{K}$ and $\mathrm{II}_{1}$ does not precede $\mathrm{II}_{3}$. We use this result to give another proof of Kronecker's theorem on canonical pairs of matrices under equivalence. If $R$ is a tame hereditary finite-dimensional algebra we show that there is a preorder on the family of isomorphism types of indecomposable $R$-modules of finite length that satisfies Conditions (b) and (c).
\end{abstract}

1991 Mathematics subject classification (Amer. Math. Soc.): 16 D 70, 15 A 21.

\section{Precedence relations}

With a few exceptions, all modules in this paper are unital right modules of finite length over an artinian ring $R$. Modules will often be used interchangeably with their (isomorphism) types, for example, the length of a type II is the length of a module whose type is II. A family, $S$, of types is said to be bounded $($ by $m$ ) if there is a positive integer $m$. such that the length of every type in $S$ is less than $m$. Proposition 1.1 generalizes [7, Proposition 4.7]. The proofs of both propositions are essentially the same.

(C) 1992 Australian Mathematical Society 0263-6115/92 \$A2.00+0.00 
Proposition 1.1. Suppose $\leq$ is a preorder (a reflexive and transitive relation) on a family, $\mathscr{M}$, of isomorphism types of $R$-modules of finite length, with the following properties:

(a) contains the isomorphism type of every simple $R$-module;

(b) every bounded subfamily of $\mathscr{M}$ has a smallest type;

(c) if $M$ is a nonsplit extension of a module of type $\mathrm{II}_{1}$ by a module of type $\mathrm{II}_{2}$, with $\mathrm{II}_{1}, \mathrm{II}_{2}$ in $\mathscr{M}$, then $M$ contains a submodule whose type $\mathrm{II}_{3}$ is in $\mathscr{M}$ and $\mathrm{II}_{1}$ does not precede $\mathrm{II}_{3}$.

Then every $R$-module, $V$, of finite length is a direct sum of submodules whose isomorphism types are in $\mathscr{H}$.

Proof. Let $l(V)$ denote the length of $V$. We shall prove, by induction on $l(V)$, that $V$ satisfies the conclusion of the proposition. We may assume that $V \neq 0$. So it has a nonzero simple submodule. Hence by (a) the family $S=\{\operatorname{type}(W): W \subseteq V\} \cap \mathscr{M}$ is not empty. Since $S$ is bounded by $l(V)$, there exists $\mathrm{II}_{1} \in S$ such that $\mathrm{II}_{1} \leq \mathrm{II}$ for every $\mathrm{II} \in S$. Let $X$ be a submodule of $V$ of type $\mathrm{II}_{1}$. If $X=V$, we would be done. So we may assume that $X$ is a nonzero proper submodule of $V$. Therefore, $l(V / X)<l(V)$. By the induction hypothesis,

$$
V / X=\sum{ }_{j \in J} U_{j} / X
$$

with type $\left(U_{j} / X\right) \in \mathscr{K}$. Suppose $X$ is not a direct summand of $U_{j}$. Then $U_{j}$ is a nonsplit extension of $X$ by $U_{j} / X$. So by (c), $U_{j}$ contains a submodule $Y$ (say) of type $\mathrm{II}_{3} \in \mathscr{M}$ and $\mathrm{II}_{1}$ does not precede $\mathrm{II}_{3}$. Since $Y$ is a submodule of $V, \mathrm{II}_{3} \in S$. This contradicts the choice of $\mathrm{II}_{1}$. Therefore, $X$ is a direct summand of $U_{j}$ for each $j \in J$. This implies, from (1), that $X$ is a direct summand of $V$. Applying the induction hypothesis to a direct complement of $X$ in $V$ gives us that $V$ is a direct sum of submodules whose types are in $\mathscr{M}$.

A preorder which satisfies Conditions (a), (b), and (c) of Proposition 1.1 will be called a precedence relation. We use Proposition 1.1 to give a new exposition of Kronecker's theorem.

Let $A=\left(A_{1}, A_{2}, \ldots, A_{n}\right)$ and $B=\left(B_{1}, B_{2}, \ldots, B_{n}\right)$ be two $n$-tuples of $r \times s$ matrices. The $n$-tuple $A$ is equivalent to $B$ if there are invertible matrices $P$ and $Q$ such that

$$
P A_{i} Q=B_{i}, \quad i=1, \ldots, n .
$$

We are interested in the case $n=2$. (For $n \geq 3$, see [8], and for $n=1$, see [13].) We assume that the matrices have entries in an algebraically closed field, $K$. Following [3], we replace the pairs of matrices by pairs of linear 
transformations from an $s$-dimensional vector space $V$ to an $r$-dimensional vector space $W$. By taking linear combinations, we get from each such pair of linear transformations a $K$-bilinear map, o, from $K^{2} \times V$ to $W$. By linearity, it is enough to specify the map on a basis $(a, b)$ of $K^{2}$ and on a basis of $V$. The pair $(V, W)$ together with the bilinear map is a Kronecker module. The space $W$ is called the range or target space, while $V$ is the domain space of $(V, W)$ of $(V, W)$. Kronecker modules can be considered as modules over a finite-dimensional $K$-algebra, see, for example, [7, Proposition 0.1]. (This algebra is called a Kronecker algebra.)

Let $V=(V, W)$ be a Kronecker module. Each $e \in K^{2}$ gives rise to a linear map

$$
T_{e}: V \rightarrow W, \quad T_{e}(v)=e \circ v \text { for all } v \text { in } V .
$$

A module $(X, Y)$ is a submodule of $(V, W)$ precisely when $X$ is a subspace of $V, Y$ is a subspace of $W$ and $T_{e}(X) \subset Y$ for all $e$ in $K^{2}$. A homomorphism from a module $(U, Z)$ to $(V, W)$ is a pair of linear maps $(\varphi, \psi)$ with $\varphi$ a linear map from $U$ to $V$ and $\psi$ a linear map from $Z$ to $W$ such that for each $e \in K^{2}, u \in U$, we have

$$
e \circ \varphi(u)=\psi(e \circ u) \text {. }
$$

In (4), $\circ$ on the left hand side is in $(V, W)$ while $\circ$ on the right is in $(U, Z)$.

To say that $(U, Z)$ is isomorphic to $(V, W)$ means that there is a homomorphism $(\varphi, \psi)$ from $(U, Z)$ to $(V, W)$ with $\varphi$ and $\psi$ isomorphisms. This brings us back to (2), with $n=2$, when $\varphi$ and $\psi$ are interpreted as matrices.

If $(X, Y)$ is a submodule of $(V, W)$, then $(V, W) /(X, Y)=(V / X$, $W / Y)$ is a module via

$$
e \circ(v+X)=e \circ v+Y
$$

for all $v \in V$, all $e \in K^{2}$, where $e \circ v$ is from the bilinear map in $(V, W)$.

Let $(V, W)$ be a module in which, for some $c$ in $K^{2}$, the linear map $T_{c}$ (see (3)) is an isomorphism of $V$ onto $W$. The map from $K^{2} \times V$ to $V$ that takes $(e, v)$ to $T_{c}^{-1}(e \circ v)$ is bilinear and so makes $(V, V)$ a module. Let id be the identity map on $V$. Then (id, $T_{c}^{-1}$ ) is an isomorphism from $(V, W)$ to $(V, V)$. Moreover, $V$ is a $K[\zeta]$-module, $\zeta$ an indeterminate over $K$; see, for example, [13]. Conversely, let $V$ be a $K[\zeta]$-module. We make $(V, V)$ a Kronecker module as follows. Let $(a, b)$ be a fixed basis of $K^{2}$. Given $e=\alpha a+\beta b \in K^{2}, v \in V$, set $e \circ v=(\alpha+\beta \zeta) v$. We summarize this discussion in Proposition 1.2. 
Proposition 1.2. A Kronecker module $(V, W)$ is isomorphic to a module that comes from a $K[\zeta]$-module if and only if for some $e$ in $K^{2}, T_{e}$ is an isomorphism of $V$ onto $W$.

If $(U, Z)$ is an extension of $(X, Y)$ by $(V, W)$ and $T_{e}$ is an isomorphism of $X$ onto $Y$ and $V$ onto $W$ then it is also an isomorphism of $U$ onto $Z$.

The modules in Proposition 1.2 are said to be nonsingular or regular. We can call on the results in [9] when dealing with them.

Let $\theta$ be an element of $K$. A nonsingular module $(V, V)$ is said to be a $\theta$-module if for every $v$ in $V$ there exists a positive integer $n$-depending on $v$-such that $(\zeta-\theta)^{n} v=0$. An extension of a $\theta$-module by a $\theta$-module is again a $\theta$-module.

Let $V^{*}$ be the vector space of linear functionals on a vector space, $V$. Let $(V, W)$ be a Kronecker module. Then the dual module of $(V, W)=$ $\left(W^{\star}, V^{\star}\right)$ is a Kronecker module with the bilinear map given by

$$
\left(e \circ w^{\star}\right)(v)=w^{\star}(e \circ v) \quad \text { for } e \in K^{2}, w^{\star} \in W^{\star}, \text { and } v \in V .
$$

If both $V$ and $W$ are finite-dimensional, then the double dual of $(V, W)$ is naturally isomorphic to $(V, W)$.

If $S$ is a subset of a vector space $V$, then $[S]$ will denote the subspace of $V$ spanned by $S$. The dimension of a vector space $V$ will be denoted by $\operatorname{dim} V$. Let $(P, P)$ denote the Kronecker module $(K[\zeta], K[\zeta])$ that comes from the $K[\zeta]$-module, $K[\zeta]$. For $n=1,2, \ldots$, let $P_{n}$ denote the subspace of $K[\zeta]$ spanned by polynomials of degree strictly less than $n ; P_{0}$ denotes the zero space. We have that $\left(P_{n-1}, P_{n}\right)$ is a submodule of $(P, P)$.

Definitions 1.3. (a) A module isomorphic to $\left(P_{n-1}, P_{n}\right)$ is said to be of type III $^{n}$. Its dual is said to be of type $\mathrm{I}^{n}$.

(b) A module is said to be of type $\mathrm{II}_{\infty}^{n}$ if it is isomorphic to $\left(P_{n}, P_{n+1}\right) /$ $(0,[1])$.

A module is said to be of type $\mathrm{II}_{\theta}^{n}$ if it is isomorphic to $\left(P_{n}, P_{n+1}\right) /$ $\left.\left(0,[\zeta-\theta)^{n}\right]\right)$. Modules of type $\mathrm{II}_{\theta}^{n}, \theta \in K \cup\{\infty\}$, are self-dual. If a nonzero element $e \in K^{2}$ is not a multiple of $b-\theta a$ then the map $T_{e}$ in (3) is an isomorphism between the domain and target spaces of $\mathrm{II}_{\theta}^{n}$. A change of basis of $K^{2}$ transforms $\mathrm{II}_{\infty}^{n}$ to $\mathrm{II}_{\theta}^{n}, \theta \neq \infty$.

REMARK 1.4. From the definitions of the types, we get the following.

(a) If $n \geq m$, there are monomorphisms from $\mathrm{III}^{m}$ to $\mathrm{III}^{n}$ with $\mathrm{II}_{\infty}^{n-m}$ and $\mathrm{I}_{0}^{n-m}$ as respective quotients. (The monomorphisms are respectively, the canonical injection and the pair of multiplications by $\zeta^{n-m}$.) 
(b) There is an epimorphism $(\varphi, \psi): \mathrm{II}_{\theta}^{n} \rightarrow I^{n}$ with $\varphi$ monic and $\operatorname{ker} \psi$ one-dimensional.

(c) If $n \geq m$, there is an isomorphism from $I^{n}$ to $I^{m}$.

(d) If $n \geq m$, then $\mathrm{II}_{\theta}^{m}$ is a submodule of $\mathrm{II}_{\theta}^{n}$.

EXAMPLE 1.5. We now define the following preorder on $\mathscr{M}=\mathrm{I} \cup \mathrm{II} \cup \mathrm{III}$, where

$$
\begin{aligned}
\mathrm{I} & =\left\{\mathrm{I}^{m}: m=1,2, \ldots\right\}, \\
\mathrm{II} & =\left\{\mathrm{II}_{\theta}^{m}: \theta \in K \cup\{\infty\}, m=1,2, \ldots\right\}, \\
\mathrm{III} & =\left\{\mathrm{III}^{m}: m=1,2, \ldots\right\} .
\end{aligned}
$$

(a) Every type in $I$ procedes every type in II $\cup$ III, while $I^{m} \leq I^{n}$ if and only if $m \leq n$.

(b) Every type in II precedes every type in III. For a fixed $n$, every type in $\left\{\mathbf{I I}_{\theta}^{n}: \theta \in K \cup\{\infty\}\right\}$ precedes every type in $\left\{\mathbf{I I}_{\theta}^{m}: \theta \in K \cup\{\infty\}, m \leq n\right\}$.

(c) III $^{n} \leq$ III $^{m}$ if and only if $n \geq m$.

Types III $^{1}$ and $I^{1}$ are the only isomorphism types of simple Kronecker modules. It is easy to verify that the above preorder on $\mathscr{K}$ satisfies Condition (b) of Proposition 1.1. In order to show that it is a precedence relation, we need only check Condition (c) of Proposition 1.1. We need to know for which types $\mathrm{II}_{1}, \mathrm{II}_{2}$ in $\mathscr{N}$ is $\operatorname{Ext}\left(\mathrm{II}_{2}, \mathrm{II}_{1}\right) \neq 0$. The next proposition is a special case of a formula in [16] whose easy proof belies its importance; see "Note added in proof" of [16]. One can also prove the formula for Kronecker modules using the fact that the indecomposable projective types are $\mathrm{III}^{1}$ and III $^{2}$.

Proposition 1.6. Let $(V, W)$ and $(X, Y)$ be finite-dimensional Kronecker modules. Then

(7) $\operatorname{dim} \operatorname{Ext}((V, W),(X, Y))=\operatorname{dim} \operatorname{Hom}((V, W),(X, Y))$

$$
-\operatorname{dim} V \operatorname{dim} X-\operatorname{dim} W \operatorname{dim} Y+2 \operatorname{dim} V \operatorname{dim} Y .
$$

From Proposition 1.2 and $\left[9\right.$, Section 52D], we get that $\operatorname{dim} \operatorname{Ext}\left(\left(\mathbf{I I}_{\theta}^{m}, \mathrm{II}_{\eta}^{n}\right)\right.$ is the minimum of $m$ and $n$, if $\eta=\theta$; otherwise it is 0 . By Proposition 1.6, we know $\operatorname{dim} \operatorname{Ext}((V, W),(X, Y))$ once we know $\operatorname{dim} \operatorname{Hom}((V, W)$, $(X, Y))$. In computing the latter for $\mathscr{M}$ we use, without further comment, previously verified values of the former. The next lemma is easily deduced from the definitions of the types in 1.3.

LEMma 1.7. Hom $\left(\mathrm{II}_{2}, \mathrm{II}_{1}\right)$ is 0 in the following cases:

(a) $\mathrm{II}_{2} \in I$ while $\mathrm{II}_{1} \in \mathrm{II} \cup \mathrm{III}$;

(b) $\mathrm{II}_{2} \in \mathrm{II}$ while $\mathrm{II}_{1} \in \mathrm{III}$;

(c) $\mathrm{II}_{2}$ and $\mathrm{II}_{1}$ are respectively of types $\mathrm{II}_{\theta}^{m}$ and $\mathrm{II}_{\eta}^{n}, \eta \neq \theta$. 
By duality, $\operatorname{dim} \operatorname{Hom}\left(\mathrm{I}^{n}, \mathrm{I}^{m}\right)$ and $\operatorname{dim} \operatorname{Hom}\left(\mathrm{II}_{0}^{n}, \mathrm{I}^{m}\right)$ are respectively equal to $\operatorname{dim} \operatorname{Hom}\left(\mathrm{III}^{m}, \mathrm{III}^{n}\right)$ and $\operatorname{dim} \operatorname{Hom}\left(\mathrm{III}^{m}, \mathrm{II}_{\theta}^{n}\right)$.

Proposition 1.8. (a) $\operatorname{dim} \operatorname{Hom}\left(\mathrm{III}^{n}, \mathrm{III}^{m}\right)=\max (0, m-n+1)$.

(b) $\operatorname{dim} \operatorname{Hom}\left(\mathrm{III}^{n}, \mathrm{II}_{\eta}^{m}\right)=m$.

(c) $\operatorname{dim} \operatorname{Ext}\left(\mathrm{II}_{\eta}^{n}, \mathrm{I}^{m}\right)=0$ for every $\eta \in K \cup\{\infty\}$ and every positive integer $n$.

(d) $\operatorname{dim} \operatorname{Hom}\left(\mathrm{III}^{n}, \mathrm{I}^{m}\right)=n+m-2$.

Proof. We shall use the modules described in 1.3.

(a) It follows from (4) that a homomorphism $(\varphi, \psi)$ from III $^{n}$ to $\mathrm{III}^{m}$ is given by multiplications by the polynomial $f=\psi(1)$. Therefore, the degree of $f$ must be less than $m-n+1$. So, $f=0$, if $m-n+1 \leq 0$. Conversely, the pair of multiplications given by such an $f$ form a homomorphism from III $^{n}$ to III $^{m}$.

(b) We do an induction on $n$. If $n=1$, (b) holds because the dimension is that of the target space of $\mathrm{II}_{\eta}^{m}$. For $n>1$, we have, by 1.4(a), a short exact sequence

$$
0 \rightarrow \mathrm{III}^{n-1} \rightarrow \mathrm{III}^{n} \rightarrow \mathrm{II}_{0}^{1} \rightarrow 0 .
$$

This leads to the exact sequence

$$
\operatorname{Hom}\left(\mathrm{II}_{0}^{1}, \mathbf{I I}_{\eta}^{m}\right) \rightarrow \operatorname{Hom}\left(\mathrm{III}^{n}, \mathrm{II}_{\eta}^{m}\right) \rightarrow \operatorname{Hom}\left(\mathrm{III}^{n-1}, \mathrm{II}_{\eta}^{m}\right) \rightarrow \operatorname{Ext}\left(\mathrm{II}_{0}^{1}, \mathrm{II}_{\eta}^{m}\right) .
$$

If $\eta \neq 0$, the first and last terms are zero. So the two middle terms have the same dimension. If $\eta=0$, we replace (8) by a similar short exact sequence involving $\mathrm{II}_{\infty}^{1}$ instead of $\mathrm{II}_{0}^{1}$.

(c) By duality and (b), $\operatorname{dim} \operatorname{Hom}\left(\mathrm{II}_{\eta}^{n}, \mathrm{I}^{m}\right)=n$. So, (c) follows from the formula in Proposition 1.6.

(d) From (8) we get the exact sequence

$$
0 \rightarrow \operatorname{Hom}\left(\mathrm{II}_{0}^{1}, \mathrm{I}^{m}\right) \rightarrow \operatorname{Hom}\left(\mathrm{III}^{n}, \mathrm{I}^{m}\right) \rightarrow \operatorname{Hom}\left(\mathrm{III}^{n-1}, \mathrm{I}^{m}\right) \rightarrow \operatorname{Ext}\left(\mathrm{II}_{0}^{1}, \mathrm{I}^{m}\right) .
$$

By (c) the last term is 0 ; while duality and (b) give us that $\operatorname{dim} \operatorname{Hom}\left(\mathrm{II}_{0}^{1}, \mathrm{I}^{m}\right)=$ 1. It follows that for $n>1, \operatorname{dim} \operatorname{Hom}\left(\mathrm{III}^{n}, \mathrm{I}^{m}\right)=\operatorname{Hom}\left(\mathrm{III}^{n-1}, \mathrm{I}^{m}\right)+1$. Since $\operatorname{dim} \operatorname{Hom}\left(\mathrm{III}^{1}, \mathrm{I}^{m}\right)=$ the dimension of the target space of $\mathrm{I}^{m}=m-1$, the formula follows by induction on $n$.

Using $1.7,1.8$, the intervening remarks, and 1.6, we obtain the following proposition. $(X, Y)$ has the horizontal types. 
Proposition 1.9.

$$
\operatorname{dim}(\operatorname{Ext}((V, W),(X, Y)))
$$

\begin{tabular}{|cccc|}
\hline$(V, W),(X, Y)$ & $\mathrm{I}^{m}$ & $\mathrm{II}_{\eta}^{m}$ & $\mathrm{III}^{m}$ \\
\hline $\mathrm{I}^{n}$ & $\max (0, m-n-1)$ & $m$ & $m+n$ \\
\hline $\mathrm{II}_{\theta}^{n}$ & 0 & $\min (n, m) \delta_{\theta \eta}$ & $n$ \\
\hline $\mathrm{III}^{n}$ & 0 & 0 & $\max (0, n-m-1)$ \\
\hline
\end{tabular}

\section{Canonical families have precedence relations}

The first task in this section is the completion of the verification that the preorder defined on $\mathscr{C}$ in Section 1.5 is a precedence relation. We shall need a polynomial-free description of the types. Let $(a, b)$ be a basis of $K^{2}$. An element $\theta \in K$ is said to be an eigenvalue of a Kronecker module $(V, W)$ if $(b-\theta a) \circ v=0$ for some nonzero vector $v \in V$. If $a \circ v=0$ for some nonzero vector $v \in V$, we say $\infty$ is an eigenvalue of $(V, W)$. A change of basis of $K^{2}$ results in a Möbius transform of the eigenvalues of a module.

Proposition 2.1. Let $(V, W)$ be a finite-dimensional Kronecker module. Suppose $V$ and $W$ have the same dimension. Then $(V, W)$ has an eigenvalue.

Proof. Let $(a, b)$ be any basis of $K^{2}$. If $a \circ v=0$ for some nonzero vector $v$ in $V$, then $\infty$ is an eigenvalue of $(V, W)$. So we may assume that the linear map

$$
T_{a}: V \rightarrow W, \quad T_{a}(v)=a \circ v,
$$

is an isomorphism of $V$ onto $W$. By Proposition 1.2, $(V, W)$ is isomorphic to $(V, V)$. Let the bilinear map in $(V, V)$ be denoted by $\circ_{1}$. It is given by

$$
e \circ_{1} v=T_{a}^{-1}(e \circ v) \text { for } e \in K^{2}, v \in V .
$$

Let $T_{b}: V \rightarrow V$ be the linear map given by (3) with $e=b$, that is, $T_{b}(v)=$ $b \circ v$. Since $K$ is algebraically closed, the endomorphism $T_{a}^{-1} T_{b}$ of $V$ has an eigenvector $v$ belonging to an eigenvalue $\theta \in K$. From (10) we get that $(b-\theta a) \circ_{1} v=0$. So $\theta$ is an eigenvalue of $(V, V)$. So $(V, W)$ has an eigenvalue. 
We note, from the definitions in 1.3 , that modules of respective types $\mathrm{II}_{\theta}^{n}$, $\mathrm{I}^{\mathrm{n}}$ have eigenvalues. Conversely, if a Kronecker module has an eigenvalue, then it has a submodule of type $\mathrm{II}_{\theta}^{1}$ or $\mathrm{I}^{1}$. Modules of type $\mathrm{III}^{m}$ have no eigenvalues.

Let $(\varphi, \psi):\left(P_{n-1}, P_{n}\right) \rightarrow(V, W)$ be a homomorphism. Let $\varphi\left(\zeta^{i}\right)=v_{i+1}$, $i=0, \ldots, n-2 ; \psi\left(\zeta^{i}\right)=w_{i+1}, i=0, \ldots, n-1$. Then, by the definition of a homomorphism

$a \circ v_{1}=w_{1} ; \quad b \circ v_{i}=w_{i+1}=a \circ v_{i+1}, \quad i=1, \ldots, n-2 ; \quad b \circ v_{n-1}=w_{n}$.

DEFINITION 2.2. A pair of sequences $\left(\left(v_{i}\right)_{i=1}^{n-1},\left(w_{i}\right)_{i=1}^{n}\right)$ that satisfies (11) is said to be a chain of type III $^{n}$ with respect to the basis $(a, b)$. The module $\left(V_{1}, W_{1}\right)=(\varphi, \psi) P_{n-1}$ is said to be spanned by the chain. It is, therefore, of type $11 I^{n}$ as defined in 1.3 if, in addition, $\operatorname{dim} V_{1}=n-1=\operatorname{dim} W_{1}-1$.

Chains of types $\mathrm{II}_{\theta}^{n}$ and $\mathrm{I}^{n}$ are defined in a similar manner; we use the quotient modules in 1.3. More precisely, a pair of sequences $\left(\left(v_{i}\right)_{i=1}^{n},\left(w_{i}\right)_{i=1}^{n}\right)$ is said to be a chain of type $\mathrm{II}_{\theta}^{n}$ if

$$
\begin{gathered}
b_{\theta} \circ v_{1}=0 ; \quad b_{\theta} \circ v_{i+1}=a \circ v_{i}=w_{i}, \quad i=1, \ldots, n-1, \\
a \circ v_{n}=w_{n} ; \quad b_{\theta}=b-\theta a .
\end{gathered}
$$

The submodule, $\left(V_{1}, W_{1}\right)$, of $(V, W)$ spanned by the chain (12) is of type $\mathrm{II}_{\theta}^{n}$ if $\operatorname{dim} V_{1}=\operatorname{dim} W_{1}=n$. In that case, the homomorphism $(\varphi, \psi):\left(P_{n}\right.$, $\left.P_{n+1}\right) \rightarrow\left(V_{1}, W_{1}\right)$ given by $\varphi(\zeta-\theta)^{i}=v_{n-i}, \psi(\zeta-\theta)^{i}=w_{n-i}, i=$ $0, \ldots, n-1, \psi(\zeta-\theta)^{n}=0$, induces an isomorphism from $P_{n} /\left(0,\left[(\zeta-\theta)^{n}\right]\right)$ onto $\left(V_{1}, W_{1}\right)$.

If $w_{n}$ in (12) is replaced by 0 , the resulting chain is of type $\mathrm{I}^{n}$. If $(V, W)$ is of type $I^{n}$ then $\operatorname{dim} V=n=\operatorname{dim} W+1$. A change of basis of $K^{2}$ takes $\mathrm{II}_{\theta}^{n}$ to $\mathrm{II}_{\eta}^{n}$, where $\eta$ is some Möbius transform of $\theta$. On the other hand, we show in Lemma 2.3 that if $(V, W)$ is of type III $^{m}$ with respect to a basis $(a, b)$ it remains of that type with respect to any other basis of $K^{2}$. Since $\mathrm{I}^{m}$ is the dual of III $^{m}$, the same remark applies to it.

LemMa 2.3 [3, Lemma 2.5]. Suppose that $(V, W)$ is a module of type III $^{n}$ with respect to a basis $(a, b)$. Then it is of type III $^{n}$ with respect to any other basis $(c, d)$ of $K^{2}$.

Proof. When $n=1,(V, W)=\left(0,\left[w_{1}\right]\right)$ and the basis of $K^{2}$ plays no role. Since $(V, W)$ is isomorphic to $\left(P_{n-1}, P_{n}\right)$, it is enough to prove the lemma for the latter. Recall that $a$ and $b$ act respectively as inclusion and multiplication by $\zeta$ from $P_{n-1}$ to $P_{n}$. Let $c=\alpha a+\beta b, d=\gamma a+\delta b$ for 
some $\alpha, \beta, \gamma, \delta \in K$. Put $v_{i}=(\alpha+\beta \zeta)^{n-i-1}(\gamma+\delta \zeta)^{i-1}, i=1, \ldots, n-1$; $w_{i}=(\alpha+\beta \zeta)^{n-i}(\gamma+\delta \zeta)^{i-1}, i=1, \ldots, n$. The relations (11) are immediately verified with $(c, d)$ in place of $(a, b)$. The sets $\left\{v_{1}, \ldots, v_{n-1}\right\}$, $\left\{w_{1}, \ldots, w_{n}\right\}$ are linearly independent over $K$. For let

$$
w=\sum_{l=1}^{n} \alpha_{i} w_{i}=(\gamma+\delta \zeta)^{n-1} \sum_{i=1}^{n} \alpha_{i}(\alpha+\beta \zeta)^{n-i} /(\gamma+\delta \zeta)^{n-i}
$$

Since $(c, d)$ is linearly independent, $\alpha \delta-\beta \gamma \neq 0$, and therefore the map $\zeta \mapsto(\alpha+\beta \zeta) /(\gamma+\delta \zeta)$ is a field automorphism of $K(\zeta)$. So if $w=0$, the scalars $\alpha_{i}, \ldots, \alpha_{n}$ are 0 . Similarly, $\left\{v_{1}, \ldots, v_{n-1}\right\}$ is linearly independent.

If a module is spanned by a chain of type $T$, the module need not be of type $T$ because the vectors defining the chain may not be linearly independent. However when $T$ is $I^{n}$ we have the following lemma.

LEMMA 2.4. Suppose a Kronecker module $(U, Z)$ contains a nonzero submodule spanned by a chain of type $\mathrm{I}^{n}$. Then $(U, Z)$ contains a submodule of type $\mathrm{I}^{m}$ for some positive integer $m \leq n$.

Proof. Let $m$ be the least positive integer such that $(U, Z)$ contains a nonzero submodule $\left(U^{\prime}, Z^{\prime}\right)$ spanned by a chain of type $\mathrm{I}^{m}$. So $m \leq n$. Say $U^{\prime}=\left[u_{1}, u_{2}, \ldots, u_{m}\right], Z^{\prime}=\left[z_{2}, \ldots, z_{m}\right]$ and for some basis $(c, d)$ of $K^{2}$

$c \circ u_{1}=0 ; \quad c \circ u_{i+1}=d \circ u_{i}=z_{i+1}, \quad i=1, \ldots, m-1 ; \quad d \circ u_{m}=0$.

We claim that the sets $\left\{u_{1}, u_{2}, \ldots, u_{m}\right\},\left\{z_{2}, \ldots, z_{m}\right\}$ are linearly independent. Linear dependence of the former set implies, from (13), linear dependence of the latter set. Suppose $\left\{z_{2}, \ldots, z_{m}\right\}$ is linearly dependent. Let $\ell$ be some positive integer such that, for some scalars $\alpha_{2}, \ldots, \alpha_{\ell-1}, z_{\ell}=$ $\sum_{j=2}^{\ell-1} \alpha_{j} z_{j}$. We now construct a chain of type $\mathrm{I}^{\ell-1}$. Since $\ell-1<m$, this would contradict the minimality of $m$. Let $u_{1}^{\prime}=u_{1}$. For $i=2, \ldots, \ell-1$, let

$$
u_{i}^{\prime}=u_{i}-\sum_{j=1}^{i-1} \alpha_{\ell-i+j} u_{j}
$$

Let $z_{2}^{\prime}=z_{2}$. For $i=3, \ldots, \ell-1$ let

$$
z_{i}^{\prime}=z_{i}-\sum_{j=2}^{i-1} \alpha_{\ell-i+j} z_{j}
$$


From (13), we get that $c \circ u_{1}^{\prime}=0, c \circ u_{i}^{\prime}=z_{i}^{\prime}$ and $d \circ u_{i}^{\prime}=z_{i+1}^{\prime}, i=$ $2, \ldots, \ell-2$, and $d \circ u_{\ell-1}^{\prime}=z_{\ell}-\sum_{j=2}^{\ell-1} \alpha_{j} z_{j}=0$; that is, we have a chain of type $I^{\ell-1}$. This chain spans a nonzero submodule. Indeed, if we had $u_{1}^{\prime}=u_{1}=0$, then $z_{2}=0$, and $\left(\left(u_{i}\right)_{i=2}^{m},\left(z_{i}\right)_{i=3}^{m}\right)$ would be a chain of type $I^{m-1}$ spanning a nonzero submodule.

COROllaRY 2.5. Let $(\varphi, \psi)$ be a nonzero homomorphism to any module, $(V, W)$, from a $\theta$-module $(X, X)$. If $\psi$ is not monic, but $\varphi$ is monic, then the image of $(\varphi, \psi)$ contains a submodule of type $\mathrm{I}^{m}$ for some positive integer $m$.

Proof. By Lemma 2.4, it is enough to show that the image of $(\varphi, \psi)$ has a submodule spanned by a chain of type $I^{\ell}$ for some positive integer $\ell$.

For every $x \in X$, there is a positive integer $\ell$ with $(\zeta-\theta)^{\ell} x=0$, because $(X, X)$ is a $\theta$-module. With $v_{1}=(\zeta-\theta)^{\ell-1} x$, we get as in (12), a chain of type $\mathrm{II}_{\theta}^{\ell}$. If $x \neq 0$ and $\psi(x)=0$, the image in $(V, W)$ of such a chain spans a nonzero submodule spanned by a chain of type $I^{\ell}$.

THEOREM 2.6. The preorder in Example 1.5 is a precedence relation.

Proof. Condition (c) of Proposition 1.1 is all there is left to check. let

$$
0 \rightarrow(X, Y) \stackrel{(\mu, \nu)}{\longrightarrow}(U, Z) \stackrel{(\sigma, \tau)}{\longrightarrow}(V, W) \rightarrow 0
$$

be a nonsplit extension with type $(X, Y)=\mathrm{II}_{1}, \operatorname{type}(V, W)=\mathrm{II}_{2}$ and $\mathrm{II}_{1}, \mathrm{II}_{2} \in \mathscr{M}$.

Case (i), $\mathrm{II}_{1}=\mathrm{I}^{m}$. By Proposition 1.9, $m \geq 3$ and $\mathrm{II}_{2}=\mathrm{I}^{n}, n<$ $m-1$. Let $\left(V_{1}, W_{1}\right)$ be a module of type $\mathrm{I}^{m-1}$. By Remark 1.4(c), there is a map $(\varphi, \psi)$ from $\left(V_{1}, W_{1}\right)$ onto $(V, W)$. Combining this map with the sequence (14), we get from pullback the exact sequence

$$
0 \rightarrow(X, Y) \stackrel{\left(\mu_{1}, \nu_{1}\right)}{\longrightarrow}\left(U_{1}, Z_{1}\right) \stackrel{\left(\sigma_{1}, \tau_{1}\right)}{\longrightarrow}\left(V_{1}, W_{1}\right) \rightarrow 0
$$

with a map $\left(\varphi_{1}, \psi_{1}\right):\left(U_{1}, Z_{1}\right) \rightarrow(U, Z)$ whose kernel is isomorphic to $\operatorname{ker}(\varphi, \psi)$. By Proposition 1.9, $\left(U_{1}, Z_{1}\right)$ is of type $\mathrm{I}^{m} \oplus \mathrm{I}^{m-1}$. Now, $\left(\varphi_{1}, \psi_{1}\right) I^{m-1}$ is a nonzero module spanned by a chain of type $I^{m-1}$. By Lemma 2.4, $(U, Z)$ has a submodule of type $I^{\ell}, \ell \leq m-1$. From 1.5, we see that $\mathrm{I}^{m}$ does not precede $\mathrm{I}^{\ell}$.

Case (ii), $\mathrm{II}_{1}=\mathrm{II}_{\theta}^{m}$. By Proposition 1.9, $\mathrm{II}_{2}$ is either $\mathrm{I}^{n}$ or $\mathrm{II}_{\theta}^{m}$. In the latter case, $(U, Z)$ must have a submodule of type $\mathrm{II}_{\theta}^{n+1}$, by Proposition 1.2, and Section 15 of [9]. From 1.5, we see that $\mathrm{II}_{\theta}^{m}$ does not precede $\mathrm{II}_{\theta}^{m+1}$. 
So let $(V, W)$ in (14) be of type $\mathrm{I}^{n}$. Let $\left(V_{1}, W_{1}\right)$ be a module of type $\mathrm{II}_{\theta}^{n}$. By $1.4(\mathrm{~b})$, there is an epimorphism $(\varphi, \psi):\left(V_{1}, W_{1}\right) \rightarrow(V, W)$ with $\varphi$ monic and $\psi$ has a one-dimensional kernel. Using this epimorphism, we proceed as in Case (i) to obtain (15). This time, $\left(U_{1}, Z_{1}\right)$ is a $\theta$-module. By Corollary 2.5, $(U, Z)$ has a submodule of type $\mathrm{I}^{\ell}$ for some positive integer $\ell$.

Case (iii), $\mathrm{II}_{1}=\mathrm{III}^{m}$. If $(U, Z)$ has an eigenvalue, then, as remarked after $2.1,(U, Z)$ has a submodule of type $\mathrm{II}_{\theta}^{1}$ or $\mathbf{I}^{1}$. Since these types are not preceded by III $^{m}$ we may assume that $(U, Z)$ has no eigenvalues. This precludes the possibility that $\mathrm{II}_{2}=\mathrm{I}^{n}$ because in that case $\operatorname{dim} U=\operatorname{dim} X+$ $\operatorname{dim} V=m-1+n=\operatorname{dim} Y+\operatorname{dim} Z$, which implies by Proposition 2.1 that $(U, Z)$ has an eigenvalue. If $\mathrm{II}_{2}=\mathrm{III}^{n}$, then by Proposition 1.9, $n>m+1$. By $1.4(\mathrm{a}),(V, W)$ has a submodule $\left(U_{1}, Z_{1}\right) /(X, Y)$ of type $\mathrm{III}^{m+1}$. By Proposition 1.9, we have a decomposition $\left(U_{1}, Z_{1}\right)=(X, Y) \oplus\left(U_{2}, Z_{2}\right)$ with $\left(U_{2}, Z_{2}\right) \subset\left(U_{1}, Z_{1}\right) \subset(U, Z)$ and type $\left(U_{2}, Z_{2}\right)=\mathrm{III}^{m+1}$. This is not preceded by $\mathrm{III}^{m}$. There remains the case $\mathrm{II}_{2}=\mathrm{II}_{\theta}^{n}$. By $1.4(\mathrm{~d}),(V, W)$ contains a submodule $\left(U_{1}, Z_{1}\right) /(X, Y)$ of type $\mathrm{II}_{\theta}^{1}$. The extension

$$
0 \rightarrow(X, Y) \rightarrow\left(U_{1}, Z_{1}\right) \rightarrow\left(U_{1}, Z_{1}\right) /(X, Y) \rightarrow 0
$$

does not split because otherwise $\left(U_{1}, Z_{1}\right)$ would contain a submodule of type $\operatorname{II}_{\theta}^{1}$. And so $\left(U_{1}, Z_{1}\right)$, hence $(U, Z)$, would have an eigenvalue. By Lemma 2.3 , we can describe the modules of the III $^{\ell}, \ell$ an arbitrary positive integer, using the basis $(b-\theta a, a)$ of $K^{2}$. (If $\theta=\infty$ we replace $(b-\theta a, a)$ by $(a, b)$.$) From this and 1.4(a), we get an extension$

$$
0 \rightarrow \mathrm{III}^{m} \rightarrow \mathrm{III}^{m+1} \rightarrow \mathrm{II}_{\theta}^{1} \rightarrow 0 .
$$

By Proposition 1.8(a), dim End III ${ }^{m+1}=1$. So $\mathrm{III}^{m+1}$ is indecomposable. Therefore, (17) does not split. By Proposition 1.9, $\operatorname{dim} \operatorname{Ext}\left(\mathrm{II}_{\theta}^{1}, \mathrm{III}^{m}\right)=1$. Hence the sequence (16) is a multiple of the sequence (17). Hence, the submodule $\left(U_{1}, Z_{1}\right)$ of $(U, Z)$ is of type III $^{m+1}$, which is not preceded by $\mathrm{III}^{m}$. This completes the proof of the theorem. So every finite-dimensional Kronecker module is a direct sum of modules whose types are in $\mathscr{M}=$ I $\cup$ II $\cup$ III .

Remark on Case (ii). When $(X, Y)$ and $(V, W)$ are of type $\mathrm{II}_{\theta}^{m}$ and $\mathrm{II}_{\theta}^{n}$ we referred to section 15 of [9] thereby implicitly relying on the structure of finitely generated torsion $K[\zeta]$-modules. Since the latter is part of Kronecker's theorem, it is interesting that the following argument avoids a reference to [9]. 
A nonsplit extension of $\mathrm{II}_{\theta}^{m}$ by $\mathrm{II}_{\theta}^{n}$ contains a submodule of type $\mathrm{II}_{\theta}^{n+1}$. To prove this, we may by Proposition 1.2 assume that we have the following nonsplit extension of $K[\zeta]$-modules

$$
0 \rightarrow X \rightarrow U \rightarrow V \rightarrow 0
$$

where $X \cong K[\zeta] /(\zeta-\theta)^{m}, V \cong K[\zeta] /(\zeta-\theta)^{n}$ with respective generators $x$ and $v$.

Let $u+X=v$. If $(\zeta-\theta)^{n} u=0$, then the map $p(\zeta) v \mapsto p(\zeta) u$ is well-defined and gives a splitting of (18). Since (18) does not split, we have $(\zeta-\theta)^{n} u=\alpha_{m-k}(\zeta-\theta)^{m-k} x+\cdots+\alpha_{m-1}(\zeta-\theta)^{m-1} x$, where $m-k \geq 0$ and $\alpha_{m-k} \neq 0, k \geq 1$. Therefore, $\langle u\rangle \cong K[\zeta] /(\zeta-\theta)^{n+k}$.

If $n+k \geq m+1$, then we are done. Otherwise, the element $u^{\prime}=u-$ $\left\{\alpha_{m-k}(\zeta-\theta)^{m-(n+k)} x+\cdots+\alpha_{m-1}(\zeta-\theta)^{m-(n+1)} x\right\}$ gives $\left\langle u^{\prime}\right\rangle \cong V$ and the map $v \mapsto u^{\prime}$ gives a splitting of (18). Hence $n+k \geq m+1$ as required.

REMARK 2.7. There are many other proofs of Kronecker's theorem on canonical pairs of matrices under equivalence, for example [3], [5], [6], [10], [11], [14], [16], [17], and [18]. Some applications of the theorem can be found in [1], [2], [10], and [12].

A Kronecker algebra is an example of a tame finite-dimensional hereditary algebra as defined in [15]. For the rest of the paper, $R$ is a tame finitedimensional hereditary algebra over an algebraically closed field $K$. In [15] it is shown that there are precisely three families of finite-dimensional indecomposable $R$-modules: $\mathscr{P}=\left(P_{n}\right)_{n=1}^{\infty},\left(S_{\theta}^{n}\right)_{n=1}^{\infty}$, for each $\theta \in K \cup\{\infty\}$, and $\mathscr{I}=\left(I_{n}\right)_{n=1}^{\infty}$. In [15, p. 350], it is shown that the indexing on $\mathscr{P}$ can be done to ensure that

$$
\operatorname{Hom}\left(P_{i}, P_{j}\right) \neq 0 \Rightarrow i \leq j .
$$

Similarly the indexing on $\mathscr{I}$ is chosen to have the property

$$
\operatorname{Hom}\left(I_{i}, I_{j}\right) \neq 0 \Rightarrow i \geq j \text {. }
$$

The families $\mathscr{P}$ and $\mathscr{I}$ are closed under indecomposable submodules and indecomposable quotients respectively [15, Propositions 2.7 and 3.4]. So, from (19) and (20), End $M=K$ for each $M$ with type $M \in \mathscr{P} \cup \mathscr{I}$.

Each $S_{\theta}^{n}$ may be considered as a module over a discrete valuation ring [15, Section 4] and hence amenable to the same treatment as $\mathrm{II}_{\theta}^{n}$. Corresponding to III $^{n}$ and $\mathrm{I}^{n}$ are $P_{n}$ and $\mathrm{I}_{n}$ respectively. With these correspondences in mind, we define a preorder, $\leq$, on $\mathscr{F}$, the family of isomorphism classes of finite-dimensional indecomposable $R$-modules, exactly as 1.5 . To show that $\leq$ is a precedence relation we shall proceed as in the proof of Theorem 2.6 with the simplification that we know that $\mathscr{F}$ is canonical. 
THeOREM 2.8. The family $\mathscr{F}$ of finite-dimensional indecomposable isomorphism types over a tame finite-dimensional hereditary algebra has a precedence relation.

Proof. We shall show that the preorder, defined above, on $\mathscr{F}$ is a precedence relation. Condition (b) is readily checked. To check Condition (c), we let

$$
0 \rightarrow L \rightarrow M \rightarrow N \rightarrow 0
$$

be a nonsplit sequence of $R$-modules.

Case (i), $L=\mathrm{I}_{m}$. By [15, Corollary 3.5], $N=\mathrm{I}_{n}$ for some positive integer $n$. Moreover, by [15, Proposition 3.4], $M=\bigoplus \mathrm{I}_{n_{j}}$, a finite direct sum of modules in $\mathscr{I}$. It follows from (20) and the nonsplitting of (21) that each $n_{j}<m$.

Case (ii), $L=S_{\theta}^{n}$. By [15, Section 4], $N=\mathrm{I}_{n}$ or $S_{\theta}^{n}$. The latter case is handled in the same way as the corresponding case in Theorem 2.6. If $N=\mathrm{I}_{n}$, then by [15, Proposition 4.2], $M$ must have a direct summand in $\mathscr{I}$.

Case (iii), $L=P_{m}$. If $M$ has a submodule isomorphic to $\mathrm{I}_{n}$ or $S_{\theta}^{n}$ we would be done because $P_{m}$ does not precede those types. So, by [15, Section 4.1], we may assume that $M=\bigoplus P_{n_{j}}$, a finite direct sum of modules in $\mathscr{P}$. It follows from (19) and the nonsplitting of (21) that each $n_{j}>m$.

We do not know if there are other artinian rings of infinite type for which Theorem 2.8 holds.

\section{References}

[1] N. Aronszajn, 'Quadratic forms on vector spaces', in: Proc. Intern. Symposium on Linear spaces, 1960, (Jerusalem Academic Press, Israel Academy of Sciences and Humanities, and Pergamon Press, London. Jerusalem 1961), pp. 29-87.

[2] N. Aronszajn and R. D. Brown, 'Finite-dimensional perturbations of spectral problems and variational approximation methods for eigenvalue problems I,'Studia Math. 36 (1970), 1-76.

[3] N. Aronszajn and U. Fixman, 'Algebraic spectral problems,' Studia Math. 30 (1968), 273-338.

[4] C. W. Curtis and I. Reiner, Methods of representation theory with applications to finite groups and orders, Volume I, (John Wiley and Sons, New York, 1981).

[5] L. E. Dickson, 'Singular case of bilinear, quadratic or hermitian forms,' Trans. Amer. Math. Soc. 29 (1927), 239-253.

[6] J. Dieudonné, 'Sur la réduction canonique des couples de matrices,' Bull. Soc. Math. France 74 (1946), 130-146. 
[7] U. Fixman, F. Okoh, and N. Sankaran, 'Internal functors for systems of linear transformations,' J. Algebra 113 (1988), 399-415.

[8] S. Friedland, 'Simultaneous similarity of matrices,' Advances in Math. 50 (1983), 189265.

[9] L. Fuchs, Infinite abelian groups, Volume I, Academic Press, New York, 1970.

[10] F. R. Gantmacher, Theory of matrices (Interscience, New York, 1959.)

[11] C. Greither, 'Die unzerlegbaren Moduln über $K[X, Y] /(X, Y)^{2}$,' Bericht Nr. 39, 1979, Seminar F. Kasch, B. Pareigis, Mathematisches Institut Der Universität München, Verlag Uni-Drück München.

[12] A. Heller and I. Reiner, 'Indecomposable representations,' Illinois J. Math. 5 (1961), 314-323.

[13] N. Jacobson, Lectures in abstract algebra, Volume, II Linear Algebra (D. Van Nostrand), Princeton, 1953.

[14] L. Kronecker, 'Algebraische Reduktion der Schaaren bilinearer Formen,' Sitzungsberichte Akad. Berlin (1890), 1225-1237.

[15] C. M. Ringel, 'Infinite-dimensional representations of finite-dimensional hereditary algebras,' Symposia Mat. Inst. Alta Mat. 23 (1979), 321-412.

[16] C. M. Ringel, 'Representation of $K$-species and bimodules,' J. Algebra 41 (1976), 269302.

[17] H. W. Turnbull, 'On the reduction of singular matrix pencils,' Proc. Edinburgh Math. Soc. 4 (1935), 67-76.

[18] H. W. Turnbull and A. C. Aitken, An introduction to the theory of canonical matrices (Blackie, London, 1932).

Queen's University

Kingston, Ontario K7L 3N6

Canada
Wayne State University

Detroit, Michigan 48202

USA 